\title{
Computing Power Series Solutions of a Nonlinear PDE System
}

\author{
E. Hubert \\ INRIA - projet CAFE \\ Sophia Antipolis, France \\ Evelyne.Hubert@inria.fr
}

\author{
N. Le Roux \\ LACO \\ Université de Limoges, France \\ Nicolas.Leroux@unilim.fr
}

\begin{abstract}
This paper presents a new algorithm to compute the power series solutions of a significant class of nonlinear systems of partial differential equations. The algorithm is very different from previous algorithms to perform this task. Those relie on differentiating iteratively the differential equations to get coefficients of the power series, one at a time. The algorithm presented here relies on using the linearisation of the system and the associated recurrences. At each step the order up to which the power series solution is known is doubled. The algorithm can be seen as belonging to the family of Newton iteration methods.
\end{abstract}

\section{Categories and Subject Descriptors}

I.1.2H.4 [Symbolic and Algebraic Manipulation]: Computing Methodologies-Algorithms

\section{General Terms}

Algorithms

\section{Keywords}

nonlinear partial differential systems, formal integrability, power series solutions, differential algebra, Newton iteration.

\section{INTRODUCTION}

A Newton method for computing the power series solution of a nonlinear system of ordinary differential equations of first order was introduced in [5, 8]. This paper offers a generalization to a significant class of nonlinear systems of partial differential equations.

After the work of Kowalevskaya, Riquier [21] proved the existence and uniqueness of analytic solutions to a wider class of systems of partial differential equations, the passive orthonomic systems. Riquier provided furthermore an algorithm that would bring any linear system, as well as some lucky nonlinear ones, to a system of that form. The proof

Permission to make digital or hard copies of all or part of this work for personal or classroom use is granted without fee provided that copies are not made or distributed for profit or commercial advantage and that copies bear this notice and the full citation on the first page. To copy otherwise, to republish, to post on servers or to redistribute to lists, requires prior specific permission and/or a fee.

ISSAC'03, August 3-6, 2003, Philadelphia, Pennsylvania, USA.

Copyright 2003 ACM 1-58113-641-2/03/0008 ...\$5.00. of existence and uniqueness of Riquier consists in proving first the existence and uniqueness of a formal power series solution. At a second stage the series is proved to converge. Riquier's approach was completed for polynomially nonlinear differential equations by Ritt [22] by use of characteristic set method. See also [30].

This paper first presents a general framework for power series solution of polynomially nonlinear differential systems. Though borrowing heavily from $[24,3,15]$ our presentation is more complete and compact. Proofs of existence and uniqueness of formal power series solutions indeed appear in [24] for rif' form systems and in [3, 15] for regular differential systems. Uniqueness is understood for a given regular initial condition. Rif' form systems and regular differential system are concepts which are in fact not far apart. They generalize and in some sense simplify the concept of passivity. Their significance owes to the fact that there are algorithms that will bring any polynomially nonlinear differential system into an equivalent finite set of such systems $[14,20,2,3,10,4]$. We shall review a proof of existence and uniqueness of power series solutions of a regular differential system.

The definitions of rif' form systems and regular differential systems depend on the choice of a ranking of the derivatives. The existence of formal power series solutions is independent of the ranking chosen. On the contrary, the convergence property of the found power series does. The rankings of analycity defined in [16] are shown, in that same paper, to be the only rankings for which any analytic initial condition leads to a converging power series solution.

This paper addresses the computation of the formal power series solution of a regular differential system. The proofs of existence and uniqueness of the power series solutions of [24, 3] are constructive. The underlying algorithms are based on differential reductions, so basically on differentiation of the equations, and evaluation. We propose a new algorithm of a completely different nature for the computation of the power series solution of a regular differential system. This paper is a first report on a longer research project on the efficient computation of power series solutions. We concentrate here on showing the quadratic character of our algorithm: each step nearly doubles the order up to which the power series solution is known.

The basic step of the algorithm consists in using the linearized equations at an approximation of the solution to compute an approximation of the solution at a higher order. The extra terms of the new approximation are obtained through linear recurrence equations. If one knows an 
approximation of the solution at order $\rho>r$, where $r$ is the order of the system, the linearized system will allow us to compute an approximation of the solution at order $2 \rho-r$, or $2 \rho-r+1$ if the system is of degree one in the $r^{\text {th }}$ order derivatives. The algorithm is thus quadratic in the case of first order quasi-linear differential systems. The algorithm is described for orderly rankings. It can certainly be adapted to more general rankings, like rankings defined by weights. The algorithm can also be adapted in a straightforward way for rif' form systems.

We shall report on a first implementation of our algorithm in MAPLE. The implementation is faced with the difficulty of arithmetic operations with power series. The complexity study and a more subtle implementation is subject of future research.

The paper is divided in four sections. Some preliminaries in differential algebra are presented in Section 2. The aim of the section is to recall briefly the definition of regular differential systems and settle some notations. Evolving from $[24,3,15]$ we give in Section 3 a general and compact treatment of the formal integrability of regular differential systems. Section 4 gives the heart of our method and details the new algorithm to compute the power series solution of a regular differential system. Finally in Section 5 we compare a first implementation of our algorithm to the implementations of the libraries rif [29] and diffalg [1] of MAPLE that implement the algorithms of [24] and [3].

\section{NONLINEAR DIFFERENTIAL SYSTEMS FROM AN ALGEBRAIC VIEWPOINT}

In this section we review notations for constructive differential algebra. We shall aim at giving the definition of regular differential systems, as introduced in [2]. These systems have the excellent property of making the bridge between differential algebra and polynomial algebra. That is the key to effective algorithms that is expressed by the Rosenfeld lemma. A corollary of that lemma allows us to show, in next section, the existence and uniqueness of power series solutions for regular differential systems. Regular differential systems are all the more important that we have algorithms to decompose any differential systems into regular differential systems.

We refer the reader to [12] for an expanded tutorial presentation of this material that is fully consistent with the present notations and definitions. In particular we shall denote $\llbracket \Sigma \rrbracket$ the radical differential ideal generated by $\Sigma$. The classical notation $\{\Sigma\}$ leads to too many confusions in an algorithmic context. Classical references are the books by Ritt and Kolchin [14, 22], recent research literature include $[6,30,17,18,2,20,3,24,10,4]$ while reviews and tutorials are to be found in $[13,9]$.

We consider differential rings with commuting derivations $\left\{\delta_{1}, \ldots, \delta_{m}\right\}$. We note $\Theta$ the free commutative monoid generated by that set of derivations. An element $\theta$ of $\Theta$ can be written $\theta=\delta_{1}^{\alpha_{1}} \ldots \delta_{m}^{\alpha_{m}}$ for some $\left(\alpha_{1}, \ldots, \alpha_{m}\right) \in \mathbb{N}^{m}$ and we shall write $\theta=\delta^{\alpha}$ for short. Its order is then $|\alpha|=\alpha_{1}+\ldots+\alpha_{m}$. For $r \in \mathbb{N}$ we note $\Theta_{r}$ the set of derivation operators of order $r$ or lower. $\Theta^{+}$denotes the set of derivation operators of positive order.

Given a differential ring $\mathcal{R}$ and a set of differential indeterminates $Y=\left\{y_{1}, \ldots, y_{n}\right\}, \mathcal{R} \llbracket Y \rrbracket$ denotes the ring of differential polynomials with coefficient in $\mathcal{R}$. It is the poly- nomial ring in the infinitely many variables $\Theta Y=\{\theta y, y \in$ $Y, \theta \in \Theta\}$, called the derivatives. Let $\Sigma$ be a subset of $\mathcal{R} \llbracket Y \rrbracket$. We denote respectively $(\Sigma),[\Sigma]$ and $\llbracket \Sigma \rrbracket$ the ideal, the differential ideal and the radical differential ideal generated by $\Sigma$.

$\mathcal{R} \llbracket Y \rrbracket$ is understood to be endowed with a differential ranking, or simply ranking as no confusion shall arise. For $u \in \Theta Y$, we note $\Theta Y_{<u}$ the set of derivatives that rank lower than $u$. A ranking is orderly if it satisfies $\phi y<\psi x$, for $x, y \in Y$ and $\phi, \psi \in \Theta$, as soon as $|\phi|<|\psi|$. In that case, the sets $\Theta Y_{<u}$ are finite for any $u \in \Theta Y$.

Let $p$ be a differential polynomial of $\mathcal{R} \llbracket Y \rrbracket$ that is not in $\mathcal{R}$. The leader and separant of $p$ shall be denoted by lead $(p)$ and $\operatorname{sep}(p)$. A subset $A$ of $\mathcal{R} \llbracket Y \rrbracket$ is a differential triangular set, if no element of $A$ belongs to $\mathcal{R}$ and any element of $A$ is partially reduced w.r.t. the other ones and any two distinct elements of $A$ have distinct leaders. We denote $\mathfrak{L}(A)=\operatorname{lead}(A)$ and $S_{A}=\operatorname{sep}(A)$ the sets of leaders and separants of the elements of $A$. $\Theta A_{<u}$, for $u \in \Theta Y$ denotes the set consisting of all elements of $A$ together with all their derivatives the leaders of which are of lower rank than $u$. In other words $\Theta A_{<u}=\Theta A \cap \mathcal{R}\left[\Theta Y_{<u}\right]$.

Given an element $q \in \mathcal{R} \llbracket Y \rrbracket$ there exist processes of partial reduction that compute an element $r$ partially reduced w.r.t. $A$ such that $s q \equiv r \bmod [A]$ for some $s \in S_{A}^{\infty}$.

For $a, b \in \mathcal{R} \llbracket Y \rrbracket$ s.t. lead $(a)$ and $\operatorname{lead}(b)$ have a common derivative, while they are not derivative of one another, we define $\Delta(a, b)=\operatorname{sep}(b) \psi(a)-\operatorname{sep}(a) \phi(b)$, where $\phi, \psi \in \Theta$ are s.t. $\psi(\operatorname{lead}(a))=\phi(\operatorname{lead}(b))$ is the lowest common derivative of lead $(a)$ and lead $(b)$.

Definition $2.1 A$ pair $(A, H)$ of finite subsets of $\mathcal{R} \llbracket Y \rrbracket$ is a regular differential system if

- $A$ is a d-triangular set

- $H$ is a set of nonzero differential polynomials partially reduced w.r.t. $A$

- $S_{A} \subset H^{\infty}$

- for all $a, b \in A, \Delta(a, b) \in\left(\Theta A_{<v}\right): H^{\infty}$ where $v$ is the lowest common derivative of lead $(a)$ and lead $(b)$, if such a $v$ exists.

Regular differential systems occur as outputs of triangulation decomposition algorithms. The key of the algorithms relies on the following theorem that is an adaptation of a result of Rosenfeld [23,3].

TheOREM 2.2 (Rosenfeld's lemma) Let $(A, H)$ be a regular differential system in $\mathcal{R} \llbracket Y \rrbracket$. A differential polynomial that is partially reduced w.r.t. $A$ belongs to $[A]: H^{\infty}$ if and only if it belongs to $(A): H^{\infty}$.

Let $\mathcal{R}$ be an integral ring for which we can perform arithmetic operations and derivations as well as testing if an element is zero. Then, there are algorithms [2, 3, 10, 4, 27, 12] that computes for any pair $(\Sigma, H)$ of finite sets of differential polynomials in $\mathcal{R} \llbracket Y \rrbracket$ a finite set of regular differential systems $\left(A_{1}, H_{1}\right), \ldots\left(A_{r}, H_{r}\right)$ such that the equality

$$
\llbracket \Sigma \rrbracket: H^{\infty}=\left[A_{1}\right]: H_{1}^{\infty} \cap \ldots \cap\left[A_{r}\right]: H_{r}^{\infty}
$$

holds in $\mathfrak{Q}(R) \llbracket Y \rrbracket$, where $\mathfrak{Q}(R)$ is the field of fractions of $\mathcal{R}$. A power series is a solution of the original system $\Sigma=$ $0, H \neq 0$ if and only if it is a solution of an output system 
$A_{i}=0, H_{i} \neq 0$. Given this equivalence it is no loss of generality to inspect power series solutions of regular differential systems.

\section{FORMAL INTEGRABILITY}

We define and characterize here power series solution of a differential system. We then proceed to define regular initial conditions for a regular differential system in the way of [15]. We will then give the proof of existence and uniqueness of a power series solution to a regular differential system for a given regular initial condition. That proof goes in the sense of [3]. Another proof of existence and uniqueness of power series solution for a rif' form system ${ }^{1}$ appears in [24]. Though borrowing from $[3,24,15]$, as well as taking inspiration from [26], we feel our presentation is more complete and compact than those.

\subsection{Power series}

In this section we shall establish the notations concerning power series that will be used in the rest of the paper.

We first note $\mathcal{S}=\mathcal{K}\left[\left[t_{1}, \ldots, t_{m}\right]\right]$ the power series ring in $m$ variables with coefficients in a field $\mathcal{K}$ that is an extension of $\mathbb{Q}$, the field of rational numbers. We make it a differential ring for the derivations $\delta_{1}, \ldots, \delta_{m}$ in the usual way: $\delta_{i} t_{j}$ is equal to 1 or 0 according to whether $i=j$ or not.

We shall note $\preceq$ the partial product order on $\mathbb{N}^{m}$. The quadrant $\theta+\mathbb{N}^{m}, \theta \in \mathbb{N}^{m}$, is the set of elements $\beta \in \mathbb{N}^{m}$ s.t. $\theta \preceq \beta$. For $\alpha=\left(\alpha_{1}, \ldots, \alpha_{m}\right) \in \mathbb{N}^{m}$ we shall note $t^{\alpha}$ the term $t_{1}^{\alpha_{1}} \ldots t_{m}^{\alpha_{m}}$ and likewise $\delta^{\alpha}$ the derivation operator $\delta_{1}^{\alpha_{1}} \ldots \delta_{m}^{\alpha_{m}}$. For $\alpha, \beta \in \mathbb{N}^{m}$ we note $\alpha !=\alpha_{1} ! \ldots \alpha_{m}$ ! and if $\alpha \preceq \beta\left(\begin{array}{c}\beta \\ \alpha\end{array}\right)=\frac{\beta !}{\alpha !(\beta-\alpha) !}$.

For $s \in \mathcal{S}$ we shall note $\Phi_{\alpha}(s)$ the evaluation at $t=0$ of $\delta^{\alpha} s$ so that one can write $s=\sum_{\beta \in \mathbb{N} m} \Phi_{\beta}(s) \frac{t^{\beta}}{\beta !} . \Phi_{0}: \mathcal{S} \rightarrow \mathcal{K}$ is a ring morphism. Note that for $\alpha, \beta \in \mathbb{N}^{m}, \Phi_{\beta}\left(\delta^{\alpha} s\right)=$ $\Phi_{\alpha+\beta}(s)$ so that $\Phi_{\beta}=\Phi_{0} \circ \delta^{\beta}$. For $s, s^{\prime} \in \mathcal{S}$

$$
\Phi_{\beta}\left(s s^{\prime}\right)=\sum_{\alpha \preceq \beta}\left(\begin{array}{c}
\beta \\
\alpha
\end{array}\right) \Phi_{\beta-\alpha}(s) \Phi_{\alpha}\left(s^{\prime}\right) .
$$

\subsection{Power series solution}

In this section we inspect the meaning of power series solutions for a differential system. Usually a zero of a differential system $(\Sigma, H)$ in some differential polynomial ring $\mathcal{F} \llbracket y_{1}, \ldots, y_{n} \rrbracket$, where $\mathcal{F}$ is a differential field, is defined by a pair $\left(\mathcal{F}^{\prime}, \Psi\right)$ where $\mathcal{F}^{\prime}$ is a differential field extension of $\mathcal{F}$ and $\Psi: \mathcal{F} \llbracket y_{1}, \ldots, y_{n} \rrbracket \rightarrow \mathcal{F}^{\prime}$ is a differential ring morphism s.t. $\Psi(p)=0$ for all $p \in \Sigma$ and $\Psi(h) \neq 0$ for all $h \in H$. We adapt that definition for power series solutions.

The differential systems we shall deal with may have their coefficients in a ring of power series $\mathcal{K}_{0}\left[\left[t_{1}, \ldots, t_{m}\right]\right]$ but more usually have them in a polynomial ring $\mathcal{K}_{0}\left[t_{1}, \ldots, t_{m}\right]$. In both cases we can consider them as differential systems with coefficients in $\mathcal{S}=\mathcal{K}\left[\left[t_{1}, \ldots, t_{m}\right]\right]$ where $\mathcal{K}$ is a field extension of $\mathcal{K}_{0}$. In practice we take $\mathcal{K}$ to contain the coefficients of the initial conditions. We can indeed embed $\mathcal{K}_{0}\left[\left[t_{1}, \ldots, t_{m}\right]\right]$ and $\mathcal{K}_{0}\left[t_{1}, \ldots, t_{m}\right]$ in $\mathcal{S}$. In the case of $\mathcal{K}_{0}\left[\left[t_{1}, \ldots, t_{m}\right]\right]$ the $t_{i}$ are left invariant through the embedding while in the case of $\mathcal{K}_{0}\left[t_{1}, \ldots, t_{m}\right]$, the image of $\left(t_{1}, \ldots, t_{m}\right)$ by the embedding is $\left(a_{1}+t_{1}, \ldots, a_{m}+t_{m}\right)$ for some $\left(a_{1}, \ldots, a_{m}\right) \in \mathcal{K}^{m}$ that is the point at which we look for the power series solution.

\footnotetext{
${ }^{1}$ It is not too hard to bring a regular differential system to
} a rif' form.
That construction allows us to only consider formal power series solutions around the origin with no further extension of the base field.

Definition $3.1 A$ power series solution of the differential system $(\Sigma, H)$ of $\mathcal{S} \llbracket y_{1}, \ldots, y_{n} \rrbracket$ is defined by a morphism of differential $\mathcal{S}$-algebra $\Psi: \mathcal{S} \llbracket y_{1}, \ldots, y_{n} \rrbracket \rightarrow \mathcal{S}$ such that $\Psi(p)=0$ for all $p \in \Sigma$ while $\Psi(h) \neq 0$ for all $h \in H$.

Consider a differential ring morphism $\Psi: \mathcal{S} \llbracket y_{1}, \ldots, y_{n} \rrbracket \rightarrow$ $\mathcal{S}$. It induces the ring morphism $\bar{\Psi}=\Phi_{0} \circ \Psi$ from $\mathcal{S} \llbracket y_{1}, \ldots, y_{n} \rrbracket$ to $\mathcal{K}$ s.t. $\bar{\Psi}\left(\delta^{\theta} y_{i}\right)=\Phi_{\theta}\left(\tilde{y}_{i}\right)$, where $\tilde{y}_{i}=\Psi\left(y_{i}\right)$. If $I$ is a differential ideal of $\mathcal{S} \llbracket y_{1}, \ldots, y_{n} \rrbracket$ s.t. $I \subset \operatorname{ker} \Psi$ then $I \subset \operatorname{ker} \bar{\Psi}$.

Conversely, consider a ring morphism $\bar{\Psi}: \mathcal{S} \llbracket y_{1}, \ldots, y_{n} \rrbracket \rightarrow$ $\mathcal{K}$ and define the application $\Psi: \mathcal{S} \llbracket y_{1}, \ldots, y_{n} \rrbracket \rightarrow \mathcal{S}$ by

$$
\Psi(p)=\sum_{\beta \in \mathbb{N}^{m}} \bar{\Psi}\left(\delta^{\beta} p\right) \frac{t^{\beta}}{\beta} \text { for all } p \in \mathcal{S} \llbracket y_{1}, \ldots, y_{n} \rrbracket .
$$

One easily checks that $\Psi$ is a differential morphism leaving $\mathcal{S}$ invariant. It is thus the differential morphism over $\mathcal{S}$ defined by $\Psi\left(y_{i}\right)=\sum_{\beta \in \mathbb{N} m} \bar{\Psi}\left(\delta^{\beta} y_{i}\right) \frac{t^{\beta}}{\beta !}$. Furthermore $\Psi(p)=0$ iff $[p] \subset \operatorname{ker} \bar{\Psi}$. If $I$ is a differential ideal of $\mathcal{S} \llbracket y_{1}, \ldots, y_{n} \rrbracket$ s.t. $I \subset \operatorname{ker} \bar{\Psi}$ then $I \subset \operatorname{ker} \Psi$.

We summarize the previous discussion in a property. That shall allow us to define initial conditions and prove the existence and uniqueness of power series solutions to regular differential systems.

Proposition 3.2 In $\mathcal{S} \llbracket y_{1}, \ldots, y_{n} \rrbracket$ consider the differential system $(\Sigma, H)$. Let $\bar{\Psi}: \mathcal{S} \llbracket y_{1}, \ldots, y_{n} \rrbracket \rightarrow \mathcal{K}$ be a ring morphism s.t. $[\Sigma] \subset \operatorname{ker} \bar{\Psi}$ but $[h] \not \subset \operatorname{ker} \bar{\Psi}$, for all $h \in H$. Then the differential ring morphism $\Psi: \mathcal{S} \llbracket y_{1}, \ldots, y_{n} \rrbracket \rightarrow \mathcal{S}$ defined by

$$
\Psi\left(y_{i}\right)=\sum_{\beta \in \mathbb{N} m} \bar{\Psi}\left(\delta^{\beta} y_{i}\right) \frac{t^{\beta}}{\beta !}
$$

is a power series solution of $(\Sigma, H)$.

By extension, we shall say in this section that $\bar{\Psi}$ is a power series solution of $(\Sigma, H)$.

\subsection{Initial conditions and associated power se- ries solution.}

In this section we reproduce the definition of initial condition of regular differential system by [15] and obtain a formal integrability statement as a corollary of Rosenfeld lemma. Basically an initial condition for a d-triangular set $A$ is defined by a set of values given to the derivatives that are not proper derivatives of the leaders of $A$.

Definition 3.3 Let $(A, H)$ be a regular differential system of $\mathcal{S} \llbracket y_{1}, \ldots, y_{n} \rrbracket$. A regular initial condition for $(A, H)$ is a $\mathcal{K}$-morphism $\Psi_{0}: \mathcal{S}\left[\Theta Y \backslash \Theta^{+} \mathfrak{L}(A)\right] \rightarrow \mathcal{K}$, such that $(A) \subset$ $\operatorname{ker} \Psi_{0}$ while $\Psi_{0}(h) \neq 0$, for any $h \in H$.

An initial condition is thus defined by attributing values to the derivatives that are not proper derivative of the leaders. Checking that an initial condition is regular consists in checking that the values make the elements of $A$ vanish while making no element of $H$ vanish. This is a test on a finite number of values, the ones corresponding to the derivatives present in the elements of $A$ and $H$. Note that 
any element of $\Psi_{0}(\mathfrak{L}(A))$ is thus (separably) algebraic over $\Psi_{0}(\Theta Y \backslash \Theta \mathfrak{L}(A))$.

To an initial condition $\Psi_{0}$ we can thus associate the power series $\omega \in \mathcal{S}^{n}$ such that

$$
\omega_{i}=\sum_{\delta^{\beta} y_{i} \notin \Theta+\mathfrak{L}(A)} \Psi_{0}\left(\delta^{\beta} y_{i}\right) \frac{t^{\beta}}{\beta !} .
$$

Proposition 3.4 Let $(A, H)$ be a regular differential system of $\mathcal{S} \llbracket y_{1}, \ldots, y_{n} \rrbracket$ and $\Psi_{0}: \mathcal{S}\left[\Theta Y \backslash \Theta^{+} \mathfrak{L}(A)\right] \rightarrow \mathcal{K}$ a regular initial condition for it. There exists a unique power series solution $\Psi: \mathcal{S} \llbracket y_{1}, \ldots, y_{n} \rrbracket \rightarrow \mathcal{K}$ of $(A, H)$ extending $\Psi_{0}$. That power series solution is s.t. $\Psi(h) \neq 0$ for any $h \in H$.

Proof. We define, for any $p \in \mathcal{S} \llbracket y_{1}, \ldots, y_{n} \rrbracket, \Psi(p)$ to be $\frac{\Psi_{0}(r)}{\Psi_{0}(h)}$ for any $r \in \mathcal{S}\left[\Theta Y \backslash \Theta^{+} \mathfrak{L}(A)\right]$ and $h \in H^{\infty}$ such that $h p \equiv r \bmod [A]$. Such a pair $(h, r)$ exists and can be found by partial reduction. Furthermore, the definition does not depend on the pair $(h, r)$. Indeed if $\left(h^{\prime}, r^{\prime}\right)$ satisfies the same congruence then $h^{\prime} r-h r^{\prime} \in[A] \cap \mathcal{S}\left[\Theta Y \backslash \Theta^{+} \mathfrak{L}(A)\right]$. By Theorem 2.2, $h^{\prime} r-h r^{\prime} \in(A): H^{\infty}$ where $(A): H^{\infty}$ is viewed as an ideal in $\mathcal{S}\left[\Theta Y \backslash \Theta^{+} \mathfrak{L}(A)\right]$. There thus exists $k \in H^{\infty}$ s.t. $k\left(h^{\prime} r-h r^{\prime}\right) \in(A)$ so that $\Psi_{0}\left(h^{\prime} r-h r^{\prime}\right)=0$. Indeed $\Psi_{0}(k) \neq 0$ while $\Psi_{0}\left(k\left(h^{\prime} r-h r^{\prime}\right)\right)=0$ by definition of regular initial condition. Consequently $\frac{\Psi_{0}\left(r^{\prime}\right)}{\Psi_{0}\left(h^{\prime}\right)}=\frac{\Psi_{0}(r)}{\Psi_{0}(h)}$.

We show that the defined application $\Psi$ is a ring morphism. For $f, g \in \mathcal{S} \llbracket y_{1}, \ldots, y_{n} \rrbracket$, let $h, h^{\prime} \in H^{\infty}, r, r^{\prime} \in$ $\mathcal{S}\left[\Theta Y \backslash \Theta^{+} \mathfrak{L}(A)\right]$ be such that $h f \equiv r \bmod [A]$ and $h^{\prime} g \equiv r^{\prime}$ $\bmod [A]$. As $h h^{\prime}(f+g) \equiv h^{\prime} r+h r^{\prime} \bmod [A]$ and $h h^{\prime} f g \equiv$ $r r^{\prime} \bmod [A]$ we have $\Psi(f+g)=\frac{\Psi_{0}\left(h^{\prime} r+h r^{\prime}\right)}{\Psi_{0}\left(h h^{\prime}\right)}=\frac{\Psi_{0}(r)}{\Psi_{0}(h)}+$ $\frac{\Psi_{0}\left(r^{\prime}\right)}{\Psi_{0}\left(h^{\prime}\right)}=\Psi(f)+\Psi(g)$ and $\Psi(f g)=\frac{\Psi_{0}\left(r r^{\prime}\right)}{\Psi_{0}\left(h h^{\prime}\right)}=\frac{\Psi_{0}(r)}{\Psi_{0}(h)} \frac{\Psi_{0}\left(r^{\prime}\right)}{\Psi_{0}\left(h^{\prime}\right)}=$ $\Psi(f) \Psi(g)$ since $\Psi_{0}$ is a ring morphism.

The morphism $\Psi$ clearly extends $\Psi_{0}$. In particular for any element $h \in H$ we have $\Psi(h)=\Psi_{0}(h) \neq 0$. Now, for $p \in[A] \subset[A]: H^{\infty}$ we have $\Psi(p)=0$. Indeed, if $(h, r)$ is the pair used to define $\Psi(p)$ then by Theorem $2.2 r \in(A): H^{\infty}$. There thus exists $k \in H^{\infty}$ s.t. $k r \in(A)$ so that $h k p \equiv 0$ $\bmod [A]$. Existence is thus secured.

Assume now $\Psi: \mathcal{S} \llbracket y_{1}, \ldots, y_{n} \rrbracket \rightarrow \mathcal{K}$ is any power series solution of $(A, H)$ extending $\Psi_{0}$. For any $p \in \mathcal{S} \llbracket y_{1}, \ldots, y_{n} \rrbracket$ there exists $h \in H^{\infty}$ and $r \in \mathcal{S}\left[\Theta Y \backslash \Theta^{+} \mathfrak{L}(A)\right]$ such that $h p \equiv r \bmod [A] . \quad$ As $[A] \subset \operatorname{ker} \Psi, \Psi(h) \Psi(p)=\Psi(r)$ so that $\Psi(p)=\frac{\Psi_{0}(r)}{\Psi_{0}(h)}$ since $\Psi_{0}(h) \neq 0$. Uniqueness is thus secured too.

Note that the condition $\Psi(h) \neq 0$ satisfied by the power series solution we exhibited is more constraining than the general requirement $[h] \not \subset \operatorname{ker} \Psi$. With this process we do not obtain all the power series solution of $(A, H)$. That is an undecidable problem already in the case of ordinary differential equations [7, 19].

The above proof is constructive and discloses an algorithm, based on differentiating the equations, to compute the power series solution up to a certain order. Variants of it are implemented within the MAPLE libraries diffalg [1] and rif [29]. In next section we present a very different way of proceeding for the computation.

\section{COMPUTING POWER SERIES SOLUTIONS}

Now that we secured the existence and uniqueness of a power series of a regular differential system we proceed to present a new algorithm to compute the power series solution of a regular differential system for a given regular initial condition. The coefficients are computed inductively through recurrence relationships associated to the linearized system.

We show first that we can associate linear recurrence equations to linear differential systems in a way that the coefficients of the power series solutions of the differential systems satisfy the recurrence equations. As a result, the coefficients of the power series solutions of a linear differential system can be computed inductively.

For nonlinear differential systems the basic step of the algorithm is as follows. Assume we know the power series solution up to a certain order $\rho$. Linearize the differential system around that approximated solution. Use the associated recurrence equations to compute up to as big an order as possible the power series solution of the non-linear system. The algorithm thus belongs to a family of Newton methods.

In the case of system of ordinary differential equations (of first order) there are explicit formula for power series solutions of linear systems and those can be used to compute new coefficients of the power series solution of the non linear system $[5,8]$. No such explicit formula exists for systems of partial differential equations. More awkwardly the existence of a power series solution of the linearized systems we consider is not secured.

Let $\mathcal{R}$ be an $\mathcal{S}$-algebra. A morphism of differential $\mathcal{S}$ algebra $\Psi: \mathcal{S} \llbracket y_{1}, \ldots, y_{n} \rrbracket \rightarrow \mathcal{R}$ is completely determined by the image $\left(\tilde{y}_{1}, \ldots, \tilde{y}_{n}\right)$ of $\left(y_{1}, \ldots, y_{n}\right)$. For $p \in \mathcal{S} \llbracket y_{1}, \ldots, y_{n} \rrbracket$ we shall write $p(\tilde{y})$ for $\Psi(p)$. If $\Psi: \mathcal{S} \llbracket y_{1}, \ldots, y_{n} \rrbracket \rightarrow \mathcal{S}$ is in fact a power series solution of a differential system $(\Sigma, H)$ of $\mathcal{S} \llbracket y_{1}, \ldots, y_{n} \rrbracket$ we shall say in this section and the next that $\tilde{y}=\left(\tilde{y}_{1}, \ldots, \tilde{y}_{n}\right) \in \mathcal{S}^{n}$ is a power series solution of $(\Sigma, H)$ or simply that $\tilde{y} \in \mathcal{S}^{n}$ is a zero of $(\Sigma, H)$. Another case of interest will be the translation morphism $\tau_{\bar{y}}: \mathcal{S} \llbracket y_{1}, \ldots, y_{n} \rrbracket \rightarrow$ $\mathcal{S} \llbracket y_{1}, \ldots, y_{n} \rrbracket$, where $\bar{y}=\left(\bar{y}_{1}, \ldots, \bar{y}_{m}\right) \in \mathcal{S}^{n}$, that is defined by $\tau_{\bar{y}}\left(y_{i}\right)=\bar{y}_{i}+y_{i}$. For $p \in \mathcal{S} \llbracket y_{1}, \ldots, y_{n} \rrbracket$ we shall write $p(\bar{y}+y)$ instead of $\tau_{\bar{y}}(p)$.

\subsection{Linear differential equations}

We show that the coefficients of the power series solution of a linear differential equation can be computed inductively through a linear recurrence relationship. The order underlying the induction is dictated by the ranking $\leq$ on the differential polynomial ring.

Proposition 4.1 Assume $l$ is a linear differential polynomial of $\mathcal{S} \llbracket y_{1}, \ldots, y_{n} \rrbracket$ with leader $\delta^{\theta} y_{\kappa}$, for some $\theta \in \mathbb{N}^{m}$ and $\kappa \in\{1, \ldots, n\}$. Write it as

$$
l=\sum_{\delta^{\beta} y_{i} \leq \delta^{\theta} y_{\kappa}} a_{i \beta} \delta^{\beta} y_{i}-b \text { where } b, a_{i \beta} \in \mathcal{S} .
$$

Assume $\tilde{y} \in \mathcal{S}^{n}$ is a zero of $l$ s.t. $\Phi_{0}\left(a_{\kappa \theta}\right) \neq 0$. For any $\alpha \in \mathbb{N}^{m}, \Phi_{\theta+\alpha}\left(\tilde{y}_{\kappa}\right)$ depends linearly on a finite subset of $\left\{\Phi_{\gamma}\left(\tilde{y}_{i}\right) \mid \delta^{\gamma} y_{i}<\delta^{\theta+\alpha} y_{\kappa}\right\}$, the lower ranking coefficients. The explicit relationship is

$$
\Phi_{\alpha}(b)=\sum_{\substack{\delta^{\beta} y_{i} \leq \delta^{\theta} y_{\kappa} \\
\gamma \preceq \alpha}}\left(\begin{array}{c}
\alpha \\
\gamma
\end{array}\right) \Phi_{\alpha-\gamma}\left(a_{i \beta}\right) \Phi_{\gamma+\beta}\left(\bar{y}_{i}\right)
$$

where the coefficient of $\Phi_{\theta+\alpha}\left(\tilde{y}_{\kappa}\right)$ is $\Phi_{0}\left(a_{\kappa \theta}\right)$. 
Proof. For any $\bar{y} \in \mathcal{S}^{n}$ we have

$\Phi_{\alpha}(l(\bar{y}))=\sum_{\delta^{\beta} y_{i}<\delta^{\theta}} \sum_{y_{\kappa}}\left(\begin{array}{l}\alpha \\ \gamma\end{array}\right) \Phi_{\alpha-\gamma}\left(a_{i \beta}\right) \Phi_{\gamma}\left(\delta^{\beta} \bar{y}_{i}\right)-\Phi_{\alpha}(b)$.

As $\Phi_{\gamma}\left(\delta^{\beta} \bar{y}_{i}\right)=\Phi_{\beta+\gamma}\left(\bar{y}_{i}\right)$ and $\Phi_{\alpha}(l(\tilde{y}))=0$ we obtain the desired relationship.

Conversely, any element $\tilde{y} \in \mathcal{S}^{n}$ satisfying $\Phi_{0}\left(a_{\kappa \beta}(\tilde{y})\right) \neq 0$ and the previous relationship for any $\alpha \in \mathbb{N}^{m}$ is a series solution of $l$. As a consequence, for a regular initial condition there is a unique power series solution $\tilde{y}$ to $l$ and its missing coefficients can be computed inductively through the previous recurrence relationships. The generalization to regular differential system that are linear is not difficult (but not used in this paper).

\subsection{Linearisation}

We chose now $\leq$ to be an orderly ranking on $\mathcal{S} \llbracket y_{1}, \ldots, y_{n} \rrbracket$. We shall show that we can use the linearisation of a differential polynomial to compute extra coefficient of a power series solution of that differential polynomial.

Let $p$ be a differential polynomial of $\mathcal{S} \llbracket y_{1}, \ldots, y_{n} \rrbracket$ with leader $\delta^{\theta} y_{\kappa}, \theta \in \mathbb{N}^{m}, \kappa \in\{1, \ldots, n\}$. That implies $p \in$ $\left(\Theta_{r} Y\right)$ for any $r$ greater or equal to $|\theta|$.

Let $\bar{y}=\left(\bar{y}_{1}, \ldots, \bar{y}_{m}\right)$ be an element of $\mathcal{S}^{n}$. According to Taylor formula at order one, we can write $p(\bar{y}+y) \in$ $\mathcal{S} \llbracket y_{1}, \ldots, y_{n} \rrbracket$ as

$p(\bar{y}+y) \equiv p(\bar{y})+\sum_{\delta^{\beta} y_{i} \leq \delta^{\theta} y_{\kappa}} \frac{\partial p}{\partial \delta^{\beta} y_{i}}(\bar{y}) \delta^{\beta} y_{i} \quad \bmod \left(\Theta_{|\theta|} Y\right)^{2}$.

Let $\mathcal{L}_{p \mid \bar{y}}$ denote that linear differential polynomial in the right handside. We define a linearisation of $p$ at $\bar{y}$ and order $\rho$ to be a linear differential polynomial

$$
\mathcal{L}_{p \mid \tilde{y}}^{(\rho)}=\sum_{\delta^{\beta} y_{i} \leq \delta^{\theta} y_{\kappa}} a_{i \beta} \delta^{\beta} y_{i}+b \in \mathcal{S} \llbracket y_{1}, \ldots, y_{n} \rrbracket
$$

where $a_{i \beta} \equiv \frac{\partial p}{\partial \delta^{\beta} y_{i}}(\bar{y})$ and $b \equiv p(\bar{y}) \quad \bmod \left(t_{1}, \ldots, t_{m}\right)^{\rho}$.

For short we shall write $(t)^{\rho}$ instead of $\left(t_{1}, \ldots, t_{m}\right)^{\rho}$. If $\bar{z} \in$ $\mathcal{S}^{n}$ is such that $\bar{z} \equiv 0 \bmod (t)^{\rho}$, then $\delta^{\alpha} \bar{z} \equiv 0 \bmod (t)^{\rho-|\alpha|}$. For any $q \in\left(\Theta_{r} Y\right)^{2}$, with $r \leq \rho, q(\bar{z}) \in(t)^{2 \rho-2 r}$. Thus, if $\bar{z} \equiv 0 \bmod (t)^{\rho}$ and $\rho \geq r \geq|\theta|$ then

$$
p(\bar{y}+\bar{z}) \equiv \mathcal{L}_{p \mid \bar{y}}^{(2 \rho-2 r)}(\bar{z}) \quad \bmod (t)^{2 \rho-2 r} .
$$

Proposition 4.2 Consider $p$ in $\mathcal{S} \llbracket y_{1}, \ldots, y_{n} \rrbracket$ with leader $\delta^{\theta} y_{\kappa}$ and separant s. Let $\tilde{y} \in \mathcal{S}^{n}$ be a zero of $p$ s.t. $\Phi_{0}(s(\tilde{y})) \neq$ 0 . Let $\bar{y} \in \mathcal{S}^{n}$ be s.t. $\bar{y} \equiv \tilde{y} \bmod (t)^{\rho}$ where $\rho \geq|\theta|$. If $|\theta| \leq r \leq \rho$ then for any $\alpha \in \theta+\mathbb{N}^{m}$ s.t. $|\alpha|<2 \rho-r$, $\Phi_{\alpha}(\tilde{y})$ depends linearly on $\left\{\Phi_{\gamma}\left(\tilde{y}_{i}\right) \mid \delta^{\gamma} y_{i}<\delta^{\alpha} y_{\kappa}\right\}$, the lower ranking coefficients.

If $\mathcal{L}_{p \mid \bar{y}}^{(2 \rho-2 r)}=\sum_{\delta^{\beta} y_{i}<\delta^{\theta} y_{\kappa}} a_{i \beta} \delta^{\beta} y_{i}-b$ is a linearisation of $p$ at $\bar{y}$ and order $2 \rho-2 r$ then the explicit relationship is

$$
\Phi_{\alpha-\theta}(b)=\sum_{\substack{\delta^{\beta} y_{i} \leq \delta^{\theta} y_{\kappa} \\
\gamma \preceq \alpha-\theta}}\left(\begin{array}{c}
\alpha-\theta \\
\gamma
\end{array}\right) \Phi_{\alpha-\theta-\gamma}\left(a_{i \beta}\right) \Phi_{\gamma+\beta}\left(\tilde{y}_{i}\right)
$$

where the coefficient of $\Phi_{\alpha}\left(\tilde{y}_{\kappa}\right)$ in the right hand side is $\Phi_{0}\left(a_{\kappa \theta}\right)=\Phi_{0}(s(\bar{y})) \neq 0$.
Proof. As $\tilde{y}-\bar{y} \equiv 0 \bmod (t)^{\rho}$ and $|\alpha-\theta|<2 \rho-2 r$ we have

$$
\Phi_{\alpha-\theta}\left(\mathcal{L}_{p \mid \bar{y}}^{(2 \rho-2 r)}(\tilde{y}-\bar{y})\right)=\Phi_{\alpha-\theta}(p(\tilde{y}))=0 .
$$

Note that $a_{\kappa \theta}=s(\bar{y})$ and therefore $\Phi_{0}\left(a_{\kappa \theta}\right)=\Phi_{0}(s(\bar{y}))$. Since $\rho \geq|\theta|$ and $\bar{y} \equiv \tilde{y} \bmod (t)^{\rho}$ we have $\Phi_{0}(s(\bar{y}))=$ $\Phi_{0}(s(\tilde{y})) \neq 0$.

Making explicit the coefficient $\Phi_{\alpha-\theta}\left(\mathcal{L}_{p \mid \bar{y}}^{(2 \rho-2 r)}(\tilde{y}-\bar{y})\right)$ as we did in the proof of Proposition 4.1 we obtain the desired relationship.

We could enunciate as an immediate corollary that if $\tilde{z}$ is a series solution of the linearisation $\mathcal{L}_{p \mid \bar{y}}^{(2 \rho-2 r)}$ such that

$$
\Phi_{\beta}\left(\tilde{z}_{i}\right)= \begin{cases}0 & \text { if }|\beta|<\rho \\ \Phi_{\beta}\left(\tilde{y}_{i}-\bar{y}_{i}\right) & \text { if }|\beta| \geq \rho \text { and } \delta^{\beta} y_{i} \notin \Theta\left\{\delta^{\theta} y_{\kappa}\right\}\end{cases}
$$

then $\bar{y}+\tilde{z} \equiv \tilde{y} \bmod (t)^{2 \rho-r}$. Indeed, the inductive relationships of Proposition 4.1 proves the existence and uniqueness of such a solution $\tilde{z}$. This wording is adequate for a single equation or for a system of ordinary differential equations. The benefit of such a wording is that it leaves the choice on how to compute the coefficients of the series solution of the linear equation. In $[5,8]$ the power series solution is computed from an explicit formula and its Taylor series expansion is itself evaluated by a Newton operator. The MAPLE package associated to [25] implements that approach. On the other hand the command dsolve/series of MAPLE implements the computation through the recurrences associated to the linearized differential system. For systems of partial differential equations we have to be more specific on how to compute the additional coefficients. A reason for that is that the linearized differential systems to be considered need not to admit power series solution for themselves. An example of such a situation is given below

Let $A$ be a d-triangular set of $\mathcal{S} \llbracket y_{1}, \ldots, y_{n} \rrbracket$ s.t. $r$ is the maximal order of the leaders of the elements of $A$. For $\bar{y} \in$ $\mathcal{S}^{n}$ we define the linearisation $\mathcal{L}_{A \mid \bar{y}}^{(\rho)}$ of $A$ at $\bar{y}$ and order $\rho$ to be the set of the linearisations of the elements of $A$ at $\bar{y}$ and order $\rho$. If $s(\bar{y}) \not \equiv 0 \bmod (t)^{\rho}$ for any $s \in S_{A}$ then $\mathcal{L}_{A \mid \bar{y}}^{(\rho)}$ is a linear d-triangular set with the same set of leaders as $A$.

EXAMPLE 4.3 Let $\mathcal{S}=\mathbb{Q}[[s, t]]$ and consider the regular differential system $(A, \emptyset)$ of $\mathcal{S} \llbracket y, z \rrbracket$ where $A=\left\{y_{s}-z y^{2}, y_{t}-\right.$ $\left.y, z_{t}+z, z_{s}\right\}$. By Proposition 3.4, the regular initial condition defined by $\bar{y}=1+s+t$ and $\bar{z}=1-t$ extends in a unique way to a power series solution $(\tilde{y}, \tilde{z}) \in \mathcal{S}^{2}$. We have $(\bar{y}, \bar{z}) \equiv(\tilde{y}, \tilde{z}) \bmod (s, t)^{2}$. To compute $(\tilde{y}, \tilde{z})$ at order 3 we shall use the linearisation $\mathcal{L}_{A \mid(\bar{y}, \bar{z})}^{(2)}$ of $A$ at order 2 which is $\left\{y_{s}-2(1+s) y-(1+2 s+2 t) z-2 s-t, y_{t}-y-s-t, z_{t}+z-t, z_{s}\right\}$. $\mathcal{L}_{A \mid(\bar{y}, \bar{z})}^{(2)}$ is a differential triangular set but it is not coherent. In fact $1 \in\left[\mathcal{L}_{A \mid(\bar{y}, \bar{z})}^{(2)}\right]$ so that it admits no zero of any sorts. Informally speaking though, the incoherence in the linearized system appears only at order bigger than 2 .

We can improve Proposition 4.2 if the differential polynomial has some linearity in it. If the differential polynomial $p$ is of degree one in all its $|\theta|^{t h}$ order derivatives then

$p(\bar{y}+y) \equiv \mathcal{L}_{p \mid \bar{y}}(y) \bmod \left(\Theta_{|\theta|-1} Y\right)^{2}+\left(\Theta_{|\theta|-1} Y\right)\left(\Theta_{|\theta|} Y\right)$. so that for $\bar{z} \in \mathcal{S}^{n}$ s.t. $z \equiv 0 \bmod (t)^{\rho}$ with $\rho \geq|\theta|$

$$
p(\bar{y}+\bar{z}) \equiv \mathcal{L}_{p \mid \bar{y}}^{(2 \rho-2 r+1)}(\bar{z}) \quad \bmod (t)^{2 \rho-2 r+1} .
$$


If furthermore the differential polynomial $p$ is linear in $|\theta|^{t h}$ order derivatives then

$$
p(\bar{y}+y) \equiv \mathcal{L}_{p \mid \bar{y}}(y) \quad \bmod \left(\Theta_{|\theta|-1} Y\right)^{2} .
$$

so that $p(\bar{y}+\bar{z}) \equiv \mathcal{L}_{p \mid \bar{y}}^{(2 \rho-2 r+2)}(\bar{z}) \bmod (t)^{2 \rho-2 r+2}$. We thus obtain the following improved result in those cases.

Proposition 4.4 Additionally to the hypotheses of Proposition 4.2 assume that $p$ is of degree one in its derivatives of order $|\theta|$. Then for any $\alpha \in \theta+\mathbb{N}^{m}$ s.t. $|\alpha|<2 \rho-|\theta|+1$, $\Phi_{\alpha}(\tilde{y})$ depends linearly on $\left\{\Phi_{\gamma}\left(\tilde{y}_{i}\right) \mid \delta^{\gamma} y_{i}<\delta^{\alpha} y_{\kappa}\right\}$, the lower ranking coefficients.

If $\mathcal{L}_{p \mid \bar{y}}^{(2 \rho-2|\theta|+1)}=\sum_{\delta^{\beta} y_{i}<\delta^{\theta} y_{\kappa}} a_{i \beta}-b$ is the linearisation of $p$ at $\bar{y}$ and order $2 \rho-2|\theta|+1$ then the explicit relationship is

$$
\Phi_{\alpha-\theta}(b)=\sum_{\substack{\delta^{\beta} y_{i} \leq \delta^{\theta} y_{\kappa} \\
\gamma \preceq \alpha-\theta}}\left(\begin{array}{c}
\alpha-\theta \\
\gamma
\end{array}\right) \Phi_{\alpha-\theta-\gamma}\left(a_{i \beta}\right) \Phi_{\gamma+\beta}\left(\tilde{y}_{i}\right)
$$

where the coefficient of $\Phi_{\alpha}\left(\tilde{y}_{\kappa}\right)$ in the right hand side is $\Phi_{0}\left(a_{\kappa \theta}\right)=\Phi_{0}(s(\bar{y})) \neq 0$.

If $p$ is furthermore linear in its derivative of order $|\theta|$, we can take $|\alpha| \leq 2 \rho-|\theta|+2$ provided we consider $\mathcal{L}_{p \mid \bar{y}}^{(2 \rho-2|\theta|+2)}$.

\subsection{Algorithm}

We consider here again an orderly ranking $\leq$ on $\mathcal{S} \llbracket y_{1}, \ldots, y_{n} \rrbracket$. Let $(A, H)$ be a regular differential system in $\mathcal{S} \llbracket y_{1}, \ldots, y_{n} \rrbracket$ and $\Psi_{0}: \mathcal{S}\left[\Theta Y \backslash \Theta^{+} \mathfrak{L}(A)\right] \rightarrow \mathcal{K}$ be a regular initial condition to which we associate $\omega \in \mathcal{S}^{n}$ such that for any $i \in\{1, \ldots, n\}$

$$
\omega_{i}=\sum_{\delta^{\beta} y_{i} \notin \Theta^{+} \mathfrak{L}(A)} \Psi_{0}\left(\delta^{\beta} y_{i}\right) \frac{t^{\beta}}{\beta !} .
$$

According to Proposition $3.4 \Psi_{0}$ extends in a unique way to $\Psi: \mathcal{S} \llbracket y_{1}, \ldots, y_{n} \rrbracket \rightarrow \mathcal{K}$ that is associated to the power series solution $\tilde{y}=\sum_{\beta \in \mathbb{N} m} \Psi\left(\delta^{\beta} y\right) \frac{t^{\beta}}{\beta !}$.

We have $\omega \equiv \tilde{y} \bmod (t)^{r_{0}}$ where $r_{0}$ is the minimal order of the leaders of $A$. We want to compute, up to a certain order,

$\bar{y}_{i}=\tilde{y}_{i}-\omega_{i}=\sum_{\delta^{\beta} y_{i} \in \Theta^{+} \mathfrak{L}(A)} \Psi\left(\delta^{\beta} y_{i}\right) \frac{t^{\beta}}{\beta !}$, for all $i \in\{1, \ldots, n\}$.

Let $r$ be the maximal order of the leaders of $A$. If we have $\bar{y} \in \mathcal{S}^{n}$ s.t. $\omega+\bar{y} \equiv \tilde{y} \bmod (t)^{\rho}$ for $\rho>r$, we can compute $\bar{z} \in \mathcal{S}^{n}$ such that $\bar{z} \equiv 0 \bmod (t)^{\rho}$ and $\omega+\bar{y}+\bar{z} \equiv \tilde{y}$ $\bmod (t)^{2 \rho-2 r}$. That consists in computing the coefficients $\Phi_{\alpha}\left(\tilde{y}_{i}\right)$ s.t. $\delta^{\alpha} y_{i} \in \Theta^{+} \mathfrak{L}(A)$ and $\rho \leq|\alpha|<2 \rho-r$. The whole process relies on Proposition 4.2.

Let $a$ be an element of $A$ with leader $\delta^{\theta} y_{\kappa} \in \mathfrak{L}(A)$. Evidently $\tilde{y}$ is a zero of $a$ and $|\theta|$ is equal to $r$ or lower. According to Proposition 4.2, for any $\alpha \in \theta+\mathbb{N}^{m}$ with $|\alpha|<$ $2 \rho-r$ we can express $\Phi_{\alpha}\left(\bar{z}_{\kappa}\right)$ as a linear combination of $\left\{\Phi_{\beta}\left(\omega_{i}\right), \Phi_{\beta}\left(\bar{y}_{i}\right), \Phi_{\beta}\left(\bar{z}_{i}\right) \mid \delta^{\beta} y_{i}<\delta^{\theta} y_{\kappa}\right\}$. The coefficients of this linear combination arise from the coefficients of $\mathcal{L}_{a \mid \omega+\bar{y}}^{(2 \rho-2 r)}$. We shall in fact refer to this relationship as $\Phi_{\alpha-\theta}\left(\mathcal{L}_{a \mid \bar{y}}^{(2 \rho-2 r)}(z)\right)$ since it arises by inspecting of the coefficient of $t^{\alpha-\theta}$ in $\mathcal{L}_{a \mid \omega+\bar{y}}^{(2 \rho-2 r)}(\bar{z})$.

Take $\delta^{\alpha} y_{\kappa}$ to be the lowest derivative of order $\rho$ in $\Theta^{+} \mathfrak{L}(A)$. It is the proper derivative of the leader $\delta^{\theta} y_{\kappa}$ of an element $a$ of $A$. We can determine $\Phi_{\alpha}\left(\bar{z}_{\kappa}\right)$ thanks to the relationship $\Phi_{\alpha-\theta}\left(\mathcal{L}_{a \mid \bar{y}}^{(2 \rho-2 r)}(z)\right)$. The relationship expresses $\Phi_{\alpha}\left(\bar{z}_{\kappa}\right)$ linearly in terms of the coefficients of $\bar{y}$ and $\omega$. It is therefore completely determined. Taking then the derivatives $\delta^{\alpha} y_{\kappa}$ of $\Theta^{+} \mathfrak{L}(A)$ increasingly from there we can compute the corresponding coefficients $\Phi_{\alpha}\left(\bar{z}_{\kappa}\right)$ in terms of the coefficients of $\bar{y}, \omega$ and the previously computed coefficients of $\bar{z}$. As a result we can compute all the $\Phi_{\alpha}\left(\bar{z}_{\kappa}\right)$ that correspond to derivative $\delta^{\alpha} y_{\kappa} \in \Theta^{+} \mathfrak{L}(A)$ with $\rho \leq|\alpha|<2 \rho-r$.

Let us point out that at each step there might be distinct possible pairs $(\beta, \kappa) \in \mathbb{N}^{m} \times\{1, \ldots, n\}$ corresponding to distinct elements $a$ of $A$. As we are ensured of the existence and uniqueness of the power series solution of $(A, H)$ we are computing, any will do.

In the precise algorithm, there is an extra technical point around the fact that not all the elements of $A$ have the same order. We need to incorporate the element of $A$ order by order. An element $a$ in $A$ of order $r$ is taken in account when we have an approximation at order $r+1$ of the solution. That approximation is previously obtained thanks to the lower order equations.

Also it is possible to take $\omega \in \mathcal{S}^{n}$ without imposing that $\Phi_{\beta}\left(\omega_{i}\right)=0$ for $\delta^{\beta} y_{i} \in \Theta^{+} \mathfrak{L}(A)$. The only requirement is that $\Phi_{0}\left(a\left(\omega_{i}\right)\right)=0$ for all $a \in A$ and $\Phi_{0}\left(h\left(\omega_{i}\right)\right) \neq 0$ for all $h \in H$.

\section{Algorithm 4.5 Newton-Power-Series-Solution}

Input:

- $\mathcal{S} \llbracket y_{1}, \ldots, y_{n} \rrbracket$ endowed with an orderly ranking $\leq$

- $(A, H)$ a regular differential system in $\mathcal{S} \llbracket y_{1}, \ldots, y_{n} \rrbracket$.

- $\omega \in \mathcal{S}^{n}$ a regular initial condition for $(A, H)$

- $R$ a positive integer

Output: $\bar{y} \in \mathcal{S}^{n}$ s.t. $\bar{y} \equiv \tilde{y} \bmod (t)^{R}$ where $\tilde{y}$ is the power series solution of $(A, H)$ with initial condition $\omega$.

$$
\begin{aligned}
& r:=\text { the minimal order of the elements of } \mathfrak{L}(A) \text {; } \\
& \rho:=r+1 \text {; } \\
& \bar{y}:=0 \text {; } \\
& \text { while } \rho \leq R \text { do } \\
& B:=A \cap \mathcal{S}\left[\Theta_{r} Y\right] \\
& r_{1}:=\left\{\begin{array}{l}
R \text { if } A \backslash B=\emptyset \\
\min \{\text { ord }(u) \mid u \in \mathfrak{L}(A \backslash B)\} \text { otherwise }
\end{array}\right. \\
& \text { if each element of } B \text { is linear in the } r^{\text {th }} \text { order deriva- } \\
& \text { tives then } \\
& \rho_{1}:=\min \left\{2 \rho-r+2, r_{1}+1, R\right\} ; \\
& \text { elif each element of } B \text { is of degree one or less in the } \\
& r^{\text {th }} \text { order derivatives then } \\
& \rho_{1}:=\min \left\{2 \rho-r+1, r_{1}+1, R\right\} ; \\
& \text { else } \\
& \rho_{1}:=\min \left\{2 \rho-r, r_{1}+1, R\right\} ; \\
& \text { fi; } \\
& \bar{\omega}:=\omega \bmod (t)^{\rho_{1}} ; \\
& L:=\mathcal{L}_{B \mid \bar{\omega}+\bar{y}}^{\left(\rho_{1}-r\right)} \\
& U:=\left\{u \in \Theta^{+} \mathfrak{L}(B) \text { s.t. } \rho \leq \text { ord }(u)<\rho_{1}\right\} ; \\
& \text { for } \delta^{\alpha} y_{\kappa} \in U \text { by increasing rank do } \\
& l:=\text { an element of } L \text { with leader } \delta^{\theta} y_{\kappa} \text { s.t. } \theta \prec \alpha \text {; } \\
& z_{\alpha}:=\text { the solution of } \Phi_{\alpha-\theta}(l(z))=0 \text {; }
\end{aligned}
$$




$$
\begin{aligned}
& \bar{y}:=\bar{y}+\sum_{\delta^{\alpha} y_{\kappa} \in U} z_{\alpha} \frac{t^{\alpha}}{\alpha !} ; \\
& r:=\left\{\begin{array}{l}
r \text { if } A \backslash B=\emptyset \text { or } \rho_{1}<r_{1}+1 \\
r_{1} \text { otherwise }
\end{array}\right. \\
& \rho:=\rho_{1} ; \\
& \text { od; } \\
& \text { return }(\bar{\omega}+\bar{y}) ;
\end{aligned}
$$

$B$ is the subset of $A$ consisting of the elements of $A$ of order $r$ or less and $r_{1}$ is the minimal order of the leaders of $A$ not in $B$. At some point $B=A$ and then $r_{1}$ become irrelevant. The piece of power series solution we compute, $\bar{y} \in \mathcal{S}^{n}$, is such that $\Phi_{\alpha}\left(\bar{y}_{i}\right)=0$ for $\delta^{\alpha} y_{i} \notin \Theta^{+} \mathfrak{L}(A)$.

The invariant of the while loop is

$$
I_{0}: \quad \omega+\bar{y} \equiv \tilde{y} \quad \bmod (t)^{\rho} .
$$

The invariant is true before the while loop as $\omega \equiv \tilde{y} \bmod (t)^{r+1}$ for $r$ the minimal order of the leaders of $A$. $L$ is the linearisation at $\bar{\omega}+\bar{y}$ and at order $\rho_{1}-r$ of $B$. It is a linear d-triangular set s.t. $\mathfrak{L}(L)=\mathfrak{L}(B)$. Its coefficients are used to compute the $\Phi_{\alpha}(\bar{y})$ for $\rho \leq|\alpha|<\rho_{1}$ within the for loop. Proposition 4.2 and Proposition 4.4 ensure the exactness of the computation.

\section{EXPERIMENTAL COMPARISONS}

We made a first implementation of our algorithm in MAPLE and made an experimental comparison with the libraries rif $[24,29]$ and diffalg $[3,1]$. As of in MAPLE 8 those codes deal only with initial conditions where the coefficients are indeterminates. Our code works without distinction for both numerical (with specified values) and generic (with indeterminates) initial condition. At our request A. Witkopf adapted rif' to handle numerical initial conditions. We shall report here on comparisons with generic initial conditions.

We present a selection of examples where our implementation performs better than rif and diffalg. On the first example, our algorithm performs clearly better. The results are nonetheless not clear cut in general. One has to point out that this first example is linear so that basically the power series solution is computed through the associated recurrences that are of finite length. In the other cases the arithmetic operations on power series lessens the impact of the quadratic character of the algorithm. Series computations were indeed made in the zealous way [28] that is in their polynomial form and we only used the standard algebraic operations on polynomials in MAPLE. The use of more appropriate coding of the differential polynomials (as straight line programs for instance) and of arithmetic operations and derivations on power series solution is underway. We expect to report on a more overall success soon.

We present the selected examples as systems of differential equations. One will easily recover the regular differential systems underlying those. The leaders of the differential polynomials together, together with coefficients, are in the left handsides of the equations. Initial conditions are given in Riquier's style. The library rif has a tool to produce them. We present the timings by plotting the logarithm of the computation time as function of the logarithm of the order up to which the power series solution is computed. Computation times were obtained on the machine Jules of MEDICIS (http://medicis.polytechnique.fr).
Example 1 We consider the differential system

$$
\left\{\begin{aligned}
(1+y) u_{x x} & =\left(x^{2}+x y+y^{2}\right) v_{y} \\
v_{x} & =\left(y^{2}+y+1\right) u
\end{aligned}\right.
$$

A regular initial condition at $(x, y)=(0,0)$ is provided by the assignment of values to $u, u_{y}, u_{y y}, \ldots$ and to $u, u_{x y}, u_{x y y}, \ldots$ and to $v, v_{y}, v_{y y}, \ldots$. It thus amount to have three arbitrary power series in $y$. The initial condition can be given in Riquier's style:

$$
(\bar{u}, \bar{v})=(f(y)+g(y) x, h(y)+f(0) x)
$$

where $f, g, h$ are power series in $y$. We assume they have indeterminates as coefficients.

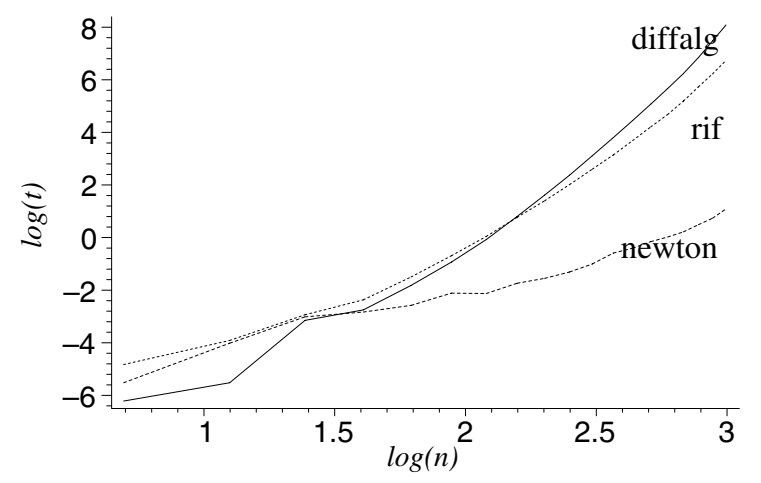

Example 2 We consider the spread disease system

$$
\left\{\begin{aligned}
v_{t} & =-r v u \\
b u_{x x} & =u_{t}-r v u+a u
\end{aligned}\right.
$$

with initial condition

$$
(\bar{u}, \bar{v})=(f(x), g(t)+h(t) x),
$$

where $f, g, h$ are power series, in $x$ or $t$ as specified within parentheses, with indeterminate coefficients.

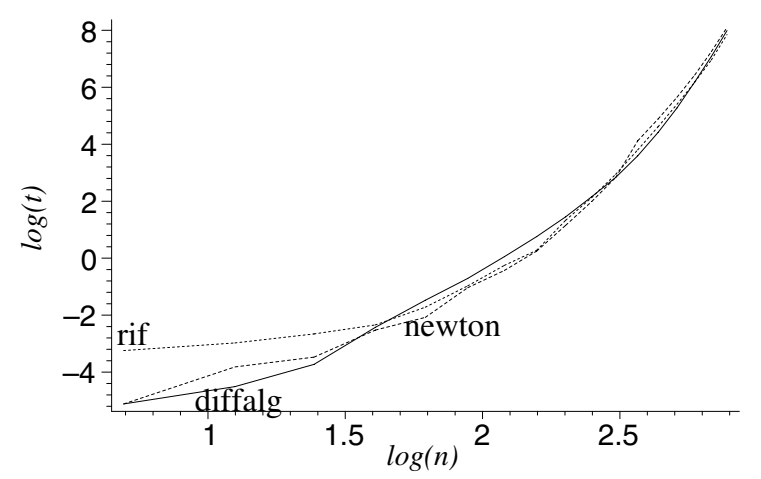

Example 3 We consider the differential equation

$$
u_{x} u_{x x}=\left(\alpha u u_{x}-\beta u_{t}\right)\left(1-t u_{x}\right)^{3}
$$

with initial condition $\bar{u}=f(t)+g(t) x+\frac{\left(\alpha f(0) g(0)-\beta f^{\prime}(0)\right)}{2 g(0)} x^{2}$, where $f$ and $g$ are power series in $t$ with indeterminate coefficients. 


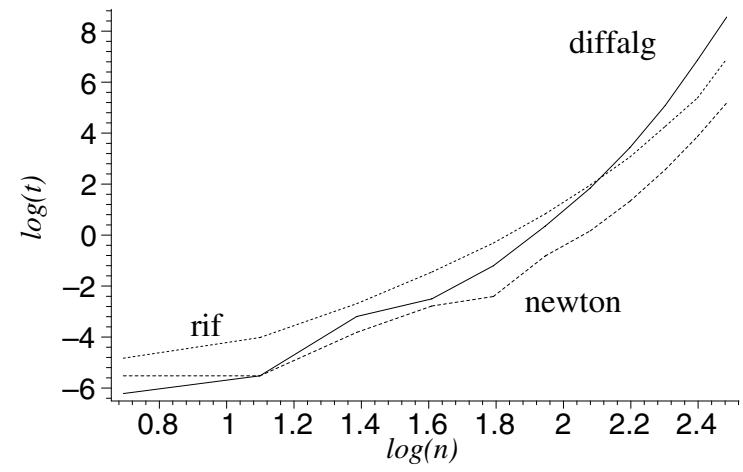

Acknowledgments: to the referees for corrections, to M. Barkatou, M. Bronstein, A. Quadrat and J-A. Weil for discussions.

\section{REFERENCES}

[1] F. Boulier and E. Hubert. diffalg: description, help pages and examples of use. Symbolic Computation Group, University of Waterloo, Ontario, Canada, 1998. Now available at http://www.inria.fr/cafe/Evelyne. Hubert/webdiffalg.

[2] F. Boulier, D. Lazard, F. Ollivier, and M. Petitot. Representation for the radical of a finitely generated differential ideal. In ISSAC. ACM Press, 1995.

[3] F. Boulier, D. Lazard, F. Ollivier, and M. Petitot. Computing representations for radicals of finitely generated differential ideals. Technical Report IT-306, LIFL, 1997.

[4] D. Bouziane, A. Kandri Rody, and H. Maârouf. Unmixed-dimensional decomposition of a finitely generated perfect differential ideal. Journal of Symbolic Computation, 31(6):631-649, 2001.

[5] R. P. Brent and H. T. Kung. Fast algorithms for manipulating formal power series. Journal of the Association Computing Machinery, 25(4):581-595, 1978.

[6] G. Carra Ferro. Gröbner bases and differential algebra. In $A A E C C$, volume 356 of Lecture Notes in Computer Science. Springer-Verlag Berlin, 1987.

[7] J. Denef and L. Lipshitz. Power series solutions of algebraic differential equations. Mathematische Annalen, 267(2):213-238, 1984.

[8] K. O. Geddes. Convergence behaviour of the Newton iteration for first-order differential equations. In Symbolic and algebraic computation, pages 189-199. Springer, Berlin, 1979.

[9] L. Guo, W. F. Keigher, P. J. Cassidy, and W. Y. Sit, editors. Differential Algebra and Related Topics. World Scientific Publishing Co., 2002.

[10] E. Hubert. Factorisation free decomposition algorithms in differential algebra. Journal of Symbolic Computation, 29(4-5):641-662, 2000.

[11] E. Hubert. Notes on triangular sets and triangulation-decomposition algorithms I: Polynomial systems. In F.Winkler and U.Langer, editors, Symbolic and Numerical Scientific Computations, LNCS. Springer, to appear.

[12] E. Hubert. Notes on triangular sets and triangulation-decomposition algorithms II: Differential systems. In F.Winkler and U.Langer, editors, Symbolic and Numerical Scientific Computating, LNCS. Springer, to appear.

[13] E. Kolchin. Selected works of Ellis Kolchin with commentary. Commentaries by Armand Borel, Michael F. Singer, Bruno Poizat, Alexandru Buium and Phyllis J. Cassidy, Edited and with a preface by Hyman Bass, Buium and Cassidy. American Mathematical Society, Providence, RI, 1999.

[14] E. R. Kolchin. Differential Algebra and Algebraic Groups, volume 54 of Pure and Applied Mathematics. Academic Press, New York-London, 1973.

[15] F. Lemaire. Contribution à l'algorithmique en algèbre différentielle. PhD thesis, Université des Sciences et Technologies de Lille, 2002.

[16] F. Lemaire. Les classements les plus généraux assurant l'analycité des solutions des systèmes orthonomes pour des conditions initiales analytiques. In CASC. Technische Universität München, 2002.

[17] E. L. Mansfield. Differential Gröbner Bases. PhD thesis, University of Sydney, 1991.

[18] F. Ollivier. Standard bases of differential ideals. In Applied algebra, algebraic algorithms and error-correcting codes (Tokyo, 1990), pages 304-321. Springer, Berlin, 1991.

[19] A. Peladan-Germa. Testing equality in differential ring extensions defined by pde's and limit conditions. Applicable Algebra in Engineering, Communication and Computing, 13(4):257-288, 2002.

[20] G. J. Reid, A. D. Wittkopf, and A. Boulton. Reduction of systems of nonlinear partial differential equations to simplified involutive forms. Eur. J. of Appl. Math., 7:604 - 635, 1996.

[21] C. Riquier. Les systèmes d'équations aux dérivées partielles. Gauthier-Villars, Paris, 1910.

[22] J. F. Ritt. Differential Algebra, volume XXXIII of Colloquium publications. American Mathematical Society, 1950. http://www.ams.org/online_bks.

[23] A. Rosenfeld. Specializations in differential algebra. Transaction of the American Mathematical Society, 90:394-407, 1959.

[24] C. J. Rust, G. J. Reid, and A. D. Wittkopf. Existence and uniqueness theorems for formal power series solutions of analytic differential systems. In ISSAC, pages 105-112. ACM, 1999.

[25] A. Sedoglavic. A probabilistic algorithm to test local algebraic observability in polynomial time. In ISSAC, pages 309-316. ACM, 2001.

[26] A. Seidenberg. Abstract differential algebra and the analytic case. Proceedings of the American Mathematical Society, 9:159-164, 1958.

[27] W. Sit. The Ritt-Kolchin theory for differential polynomials. In Guo et al. [9].

[28] J. van der Hoeven. Relax, but don't be too lazy. J. Symbolic Comput., 34(6):479-542, 2002.

[29] A. Witkopf and G. Reid. The RIF package. CECM Simon Fraser University - Vancouver, http://www.cecm.sfu.ca/ wittkopf/rif.html.

[30] W. T. Wu. On the foundation of algebraic differential geometry. Systems Science and Mathematical Sciences, 2(4):289-312, 1989. 